\title{
Modelling of straitened sedimentation process in bidisperse suspension with inter-fractional coagulation
}

\section{Tulegen Amanbaev}

South Kazakh State University, Shymkent, 5 Tauke Khanstr., 160012

Institute of Mathematics and Mathematical Modeling, Almaty, 125 Pushkinstr, Kazakhstan

\section{Email address:}

tulegen_amanbaev@mail.ru

\section{To cite this article:}

Tulegen Amanbaev. Modelling of Straitened Sedimentation Process in Bidisperse Suspension with Inter-Fractional Coagulation. American Journal of Chemical Engineering. Vol. 2, No. 2, 2014, pp. 14-20. doi: 10.11648/j.ajche.20140202.12

\begin{abstract}
In this paper we study the general characteristics of deposition of large particles (or aggregates) that result from the mutual aggregation of small and large fractions in bidisperse (or double fractional) suspension. We give equations of motion and change in mass of the large particle in the presence of inter-fractional coagulation process and effect of straitened sedimentation. In the limiting Stokes and Newton modes (relevant for small and large Reynolds numbers) we have movement formulas for speed of sedimentation and their analysis. Discuss some of the results of calculations obtained by numerical integration of the equations of motion of a large particle in suspension.
\end{abstract}

Keywords: Suspension, Brownian Motion, Sedimentation, Coagulation

\section{Introduction}

In various areas of chemical technology (production of catalysts, photographic materials, coagulative purification of liquids, etc.) are widespread processes of sedimentation of a solid phase. Thus quite often there are suspensions with strongly different sizes of particles (so-called, bidisperse or two-fractional suspensions). For example, in [1] it is noted that under certain conditions in disperse systems there can be a splitting of a range of particles of a disperse phase on two different scales fractions. Besides, there is a wide class of bidisperse suspensions in which particles of fine (small) fraction can be taken for a homogeneous medium in which the particle has a coarse(large) fraction (e.g., two-stage grinding coal-water slurries which are used in pipeline transport [2], two-fractional polymeric suspensions [3], etc). Earlier suspension with a twofractional disperse phase was observed in [4] to research the degree of dispersion of peat.

Nowadays, with the advent of increased interest in nanosuspensions (liquids with nano-scale particles) because of their unique properties studying of the sedimentation of the solid phase is quite important. This is due to the fact that nano-suspension is quite unstable, i.e. for various reasons the particles can coagulate and when it reaches a certain critical size begin to precipitate. The process of sedimentation in the suspension of heterogeneous particles with very different sizes is also used to produce nanocomposite materials.

Some aspects of two-fractional model of the coagulating disperse system are based on Smolukhovsky kinetic equation are discussed in [5,6], and in [7] the kinetics of sedimentation of a low-concentrated coagulating suspension is investigated.

The aim of this work is studying of influence of coagulation of particles of fine (small) and coarse (large) fractions in bidisperse suspension on dynamics of gravitational sedimentation of aggregates (appearing in suspension by the addition of small particles) for the effect of constraint.

Further, along with the term "large particle" will also use the term "aggregate".

\section{Basic Assumptions and Equations}

\subsection{The Key Assumptions}

Let suspension be Newtonian (unstructured), i.e. its rheological properties are determined by only hydrodynamic factors. Conditions for the existence of such suspensions are analyzed in detail in [2]. In relation to the large particle (aggregate) environment disperse system "liquid-fine particles" is viewed as a continuum with an effective viscosity and adduced density. In this case the influence of Brownian motion of small particles on the 
effective viscosity of the suspension is neglected. This approach is often used for the process of sedimentation slurry [2, 8, 9].

Let's consider coagulating dispersion system, which was already split into two fractions. Following [5], we make the following assumptions: the particles of different fractions vary considerably in size; aggregates grows by the addition of fine (small) particles; the process of coagulation growth of fine particles to aggregate size has a negligible rate compared to the direct exchange of material between the two fractions. We assume that the process of interfractional coagulation is due to two main mechanisms: Brownian motion of fine (small) particles and their engagement by large particles because of the speed difference of fine and coarse fractions of the dispersed phase of the suspension. Aggregates consist of fine particles (intervals are filled with liquid), have a spherical form and aren't subject to Brownian motion. Fragmentation of aggregates is not considered, in this connection be taken into account the effect of straitened sedimentation of large fraction as by capture of small particles the volume part of the aggregates can be substantial. For simplicity, we assume that the number density of large particles is constant and uniform throughout the space filled with a stationary suspension of fine particles (spatially homogeneous suspension). Under the latter assumption to describe the motion of the entire coarse fraction is sufficient to consider the equations of motion of only one large particle (taking into account the effects of other particles).

Further the bottom indexes 1, $l$ and $1 p$ are noted parameters of suspension, its liquid and fine components. Parameters of coarse fraction (aggregates) and the small particles attached to it are noted by the bottom indexes 2 and $2 p$ respectively.

\subsection{The Equations}

Thus, within the accepted assumptions the equations describing change of mass and velocity of aggregate in the field of gravity can be written down in a look (the coordinate axis is directed against gravity acceleration)

$$
\begin{gathered}
\frac{d m_{2}}{d t}=j, m_{2} \frac{d u_{2}}{d t}=f_{\mu}+f_{g}+f_{A}+f_{m}-j u_{2}, \\
f_{\mu}=-\frac{1}{2} C_{\mu} \pi r_{2}^{2} \rho_{1}^{\circ}\left|u_{2}\right| u_{2}, f_{g}=-V_{2} \rho_{2}^{\circ} g, f_{A}=V_{2} \rho_{1}^{\circ} g, \\
f_{m}=-\frac{1}{2} V_{2} \rho_{1}^{\circ} \frac{d u_{2}}{d t}, \\
j=n_{1 p} m_{p}\left(K_{B}+K_{c}\right), \rho_{1}^{\circ}=\frac{\alpha_{1 p} \rho_{p}^{\circ}+\alpha_{l} \rho_{l}^{\circ}, \rho_{2}^{\circ}=\alpha_{2 p}^{\max } \rho_{p}^{\circ}+\left(1-\alpha_{2 p}^{\max }\right) \rho_{l}^{\circ}}{\alpha_{2}=(4 / 3) \pi r_{2}^{3}, m_{2}=V_{2} \rho_{2}^{\circ}, \alpha_{1}+\alpha_{2}=1, \alpha_{1}=\alpha_{l}+\alpha_{1 p},} \\
\alpha_{2}=V_{2} n_{2}, n_{2}=\text { const },
\end{gathered}
$$

where $t$ is time, $m_{2}, V_{2}, r_{2}, u_{2}$ are mass, volume, radius

and speed of the large particle, $j$ is the intensity of the deposition of the fine fraction on a surface of large particles, $f_{\mu}, f_{g}, f_{A}, f_{m}$ are, respectively, force of viscous resistance, weight, Archimedes and the force of attached mass, $C_{\mu}$ is coefficient of resistance of a large particle, $n_{1 p}$, $m_{p}$ are numerical concentration of the fine fraction of the suspension and the mass of small particle, $\alpha_{l}, \alpha_{1 p}, \rho_{l}^{\circ}, \rho_{p}^{\circ}$ are volume fraction and the true density of the liquid and the fine fraction in the suspension, respectively, $\alpha_{1}, \rho_{1}^{\circ}$ are the volume concentration and the true density of the suspension of fine particles (a mixture of liquid and fine particles, without aggregates), $\alpha_{2}, n_{2}, \rho_{2}^{\circ}$ are the volume and numerical concentration of large particles in the slurry, and their true density, $\alpha_{2 p}^{\max }$ is the volume fraction of solids (fine particles) in the aggregate $\left(1-\alpha_{2 p}^{\max }\right.$ remained share is the liquid phase), which can usually be assumed constant, the density of large aggregate $\rho_{2}^{\circ}$ will also be constant (in particular, the random packing of spherical particles $\alpha_{2 p}^{\max } \cong 0.6$ [10]). Rate of change of volume concentration of the fine fraction is described by equation

$$
\frac{d \alpha_{1 p}}{d t}=-\alpha_{1 p} n_{2}\left(K_{B}+K_{c}\right)
$$

The frequencies of collisions of fine particles with a large particles in Brownian motion of small particles and due to the difference in the speeds of particles of different fractions are given by $[6,11]$

$$
\begin{gathered}
K_{B}=4 \pi k_{B} T\left(r_{p}+r_{2}\right) B\left(r_{p}\right), K_{c}=\pi \eta\left(r_{p}+r_{2}\right)^{2}\left|u_{2}\right|, \\
B\left(r_{p}\right)=\left(6 \pi \mu_{l} r_{p}\right)^{-1},
\end{gathered}
$$

where $k_{B}$ is the Boltzmann's constant, $T$ is temperature, $r_{p}$ is radius of small particles. Value $B\left(r_{p}\right)$ characterizes the mobility of fine particles and is determined by Stokes law.

Efficiency of catching of fine particles by aggregate due to the difference between their velocity $\eta$ can be calculated by the following formulas $[12,13]$ (taking into account that the ratio of particle sizes of fine and coarse fractions $\delta$ is sufficiently small).

$$
\begin{gathered}
\eta=\eta\left(\alpha_{1 p}, r_{2}, u_{2}\right) \cong\left\{\begin{array}{l}
3 \delta^{2} / 2, \quad \operatorname{Re}_{12} \leq 1, \\
(B-A) \operatorname{Re}_{12}+(2 A-B), \quad 1<\operatorname{Re}_{12} \leq 2, \\
\left(\frac{3}{2}+\frac{4}{15} \operatorname{Re}_{12}^{0.72}\right) \delta^{2}, \quad 2<\operatorname{Re}_{12} \leq 80, \\
\frac{E-D}{20} \operatorname{Re}_{12}+(5 D-4 E), \quad 80<\operatorname{Re}_{12} \leq 100, \\
3 \delta, \quad \operatorname{Re}_{12}>100,
\end{array}\right. \\
A \cong 3 \delta^{2} / 2, B \cong 1.94 \delta^{2}, \quad D \cong 7.75 \delta^{2}, E \cong 3 \delta, \delta=r_{p} / r_{2},
\end{gathered}
$$


where $\operatorname{Re}_{12}$ is Reynolds number of relative movement of aggregate. In the formula (3) the expressions at the top and bottom lines meet the limit viscous and potential modes of motion of the aggregate, and the expressions in the middle row - transients. Mechanism of catching occurs mainly because the fine particles have a finite size, and at the distance order of the radius of fine particle from the surface of a large particle the normal component of velocity of liquid is not zero [6].

Effect of constraint of motion of aggregates in case of the large volumetric concentration usually is accounted by correction $\psi$ in the resistance coefficient $[10,14,15]$

$$
\begin{gathered}
C_{\mu}=C_{\mu}^{\circ} \psi\left(\alpha_{2}\right), \psi\left(\alpha_{2}\right)=\left(1-\alpha_{2}\right)^{-m}, m \cong 5, \\
C_{\mu}^{\circ}=\frac{24}{\operatorname{Re}_{12}}+\frac{4.4}{\sqrt{\operatorname{Re}_{12}}}+0.42, \operatorname{Re}_{12}=\frac{2 \rho_{1}^{\circ} r_{2}\left|u_{2}\right|}{\mu_{1}} .
\end{gathered}
$$

To calculate the effective viscosity of the suspension with the fine fraction of particles it is advisable to use a Mooney's formula, which describes quite well the numerous experimental data in a wide range of volume content of the dispersed phase and has the form $[8,16,17]$

$$
\mu_{1}=\mu_{l} \exp \left(\frac{2.5 \alpha_{1 p}}{1-\alpha_{1 p} / \alpha_{1 p}^{\max }}\right)
$$

where $\alpha_{1 p}^{\max }$ is the volume part of the fine fraction of the suspension at the most dense packing of the particles (henceforth referred $\alpha_{1 p}^{\max }=0.74$ ). Coefficient 2.5 provides a transition Mooney's formula to Einstein's famous formula for small volume content of the fine fraction [10].

We define the following initial conditions for the system (1), (2):

$$
\begin{gathered}
t=0, m_{2}=m_{20}, \alpha_{1 p}=\alpha_{1 p 0}, u_{2}=0, \\
\alpha_{2}=\alpha_{20}=n_{20} V_{20} .
\end{gathered}
$$

\section{Results and Discussion}

\subsection{A Fist Integral}

It is easy to see that the system of equations (1), (2) has the first integral, which reflects the law of conservation of mass

$$
\begin{gathered}
\alpha_{1 p}+\frac{\alpha_{2}}{\theta}=\text { const } \\
\theta=\frac{\bar{\rho}_{p}^{\circ}}{\bar{\rho}_{2}^{\circ}}, \bar{\rho}_{p}^{\circ}=\frac{\rho_{p}^{\circ}}{\rho_{l}^{\circ}}, \bar{\rho}_{2}^{\circ}=\frac{\rho_{2}^{\circ}}{\rho_{l}^{\circ}}=1+\alpha_{2 p}^{\max }\left(\bar{\rho}_{p}^{\circ}-1\right),
\end{gathered}
$$

where the constant value on the right side is determined, for example, by the initial data. Equation (4) can be used, in particular, to compute the volume content of the coarse fraction and the radius of the aggregate

$$
\begin{gathered}
\alpha_{2}=\alpha_{20}+\theta\left(\alpha_{1 p 0}-\alpha_{1 p}\right) \\
\bar{r}_{2}=\frac{r_{2}}{r_{20}}=\left(\frac{\alpha_{2}}{\alpha_{20}}\right)^{1 / 3}=\left[1+\frac{\theta\left(\alpha_{1 p 0}-\alpha_{1 p}\right)}{\alpha_{20}}\right]^{1 / 3}
\end{gathered}
$$

Hence, in particular, derive an expression for the limiting or steady-state (when the fine fraction is fully joined to the large aggregates, i.e., when $\alpha_{1 p}=0$ ) and the radius of the appropriate limit the volume content of aggregates in suspension

$$
\bar{r}_{2 s}=\left(1+\frac{\alpha_{1 p 0}}{\alpha_{20}} \theta\right)^{1 / 3}=\left(\frac{\alpha_{2 s}}{\alpha_{20}}\right)^{1 / 3}, \alpha_{2 s}=\alpha_{20}+\theta \alpha_{1 p 0}
$$

With the growth $\bar{\rho}_{p}^{\circ}$ parameter $\theta$ tends to its maximum value $1 / \alpha_{2 p}^{\max }$. The corresponding maximum value $\alpha_{2 s}$ will be

$$
\alpha_{2 s}^{\max }=\alpha_{20}+\frac{\alpha_{1 p 0}}{\alpha_{2 p}^{\max }}
$$

This formula reflects the fact that the limit of volume fraction of large particles (aggregates) is always greater than the sum of the initial volume content of fine and coarse fractions of the dispersed phase (as in the aggregate there is also a liquid phase, which fills the space between the particles of aggregate). Because of the assumptions made in the framework the volume fraction of aggregates in suspension may not exceed the limit value corresponding to the most dense packing, so that must be done the relation $\alpha_{2 s}^{\max }<0.74$ or

$$
\alpha_{20}+\frac{\alpha_{1 p 0}}{\alpha_{2 p}^{\max }}<0.74
$$

When we give the value $\alpha_{1 p 0}$ and $\alpha_{20}$ we should keep in mind the obtained ratio.

\subsection{A Case of Non-Sedimentation}

In the absence of the sedimentation ( $\left.u_{2} \cong 0\right)$ consolidation of aggregates occurs due to Brownian coagulation. Such a situation can occur in those cases where, for example, aggregate sizes are sufficiently small or the particle substance density is not much different from the density of the liquid. In this case the system (1), (2) has an analytical solution that can be represented in an implicit form

$$
\begin{gathered}
\bar{t}=-\frac{1}{2} \ln \left[\frac{\varphi(z)}{\varphi\left(z_{0}\right)}\right]-\sqrt{3}\left[\omega(z)-\omega\left(z_{0}\right)\right], \\
\varphi(z)=\frac{\left[1-(1-z)^{1 / 3}\right]^{3}}{z}, \omega(z)=\operatorname{arctg}\left(\frac{2(1-z)^{1 / 3}+1}{\sqrt{3}}\right),
\end{gathered}
$$




$$
\begin{gathered}
z=\frac{\theta \alpha_{1 p}}{\alpha_{2 s}}=1-\frac{\alpha_{2}}{\alpha_{2 s}}=1-\left(\frac{\bar{r}_{2}}{\bar{r}_{2 s}}\right)^{3}, \bar{t}=\left(\frac{1}{2 \pi} \frac{k T}{\mu_{l} r_{p}} \frac{\alpha_{20} \bar{r}_{2 s}}{r_{20}^{2}}\right) t, \\
\left(z<z_{0}<1\right) .
\end{gathered}
$$

The graph of dependence $z(\bar{t})$ at different $z_{0}$ is shown in Fig. 1.

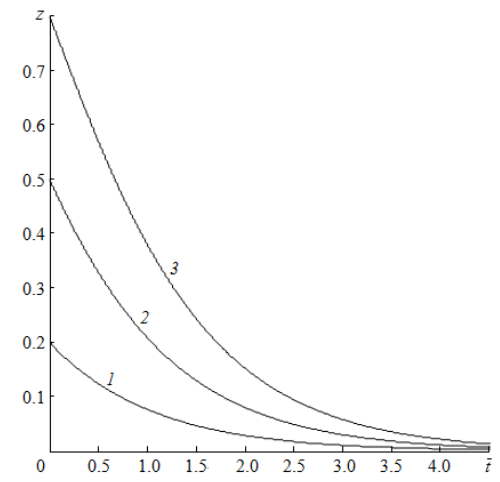

Fig. 1. Graph $z(\bar{t})$ at different $z_{0}: 1-z_{0}=0.2,2-0.5,3-0.8$.

Further it is useful to analyze the limiting Stokes and Newton regimes of the steady motion of the aggregate.

\subsection{Stokes Movement Mode of Aggregate}

In the Stokes regime, when the Reynolds number of the relative motion of large particles $\mathrm{Re}_{12}$ are small enough for the drag coefficient of the aggregate is allowed the following approximate expression $C_{\mu}^{\circ} \cong 24 / \mathrm{Re}_{12}$. In this case sedimentation rate can be expressed in the formula

$$
\bar{v}_{2 s} \equiv-\bar{u}_{2 s}=\frac{\left(\bar{\rho}_{2}^{\circ}-1\right) \bar{r}_{2 s}^{2}}{\psi\left(\alpha_{2 s}\right)}, \bar{u}_{2}=\frac{u_{2}}{U}, U=\frac{2}{9} \frac{\rho_{i}^{\circ} g r_{20}^{2}}{\mu_{l}},\left(\bar{u}_{2 s}<0, \bar{v}_{2 s}>0\right) .
$$

Analysis showed that the rate $\bar{v}_{2 s}$, defined by the formula (6) with increasing $\alpha_{20}$ monotonically decreases, whereas with a change $\alpha_{1 p 0}$ behaves not monotonically and has maximum at the following value of $\alpha_{1 p 0}$

$$
\alpha_{1 p 0}=\alpha_{1 p 0}^{*}=\frac{1}{\theta}\left(\frac{2}{17}-\alpha_{20}\right)
$$

Limit volume concentration of the coarse fraction corresponding to this value $\alpha_{1 p 0}^{*}$ is equal to

$$
\alpha_{2 s}^{*}=\alpha_{20}+\theta \alpha_{1 p 0}^{*}=\frac{2}{17} \text {. }
$$

The corresponding maximal rate of sedimentation can be calculated by the formula

$$
\bar{v}_{2 s}^{\max } \cong 0.1284 \frac{\bar{\rho}_{2}^{\circ}-1}{\alpha_{20}^{2 / 3}} .
$$

For example, for $\alpha_{20}=0.01, \alpha_{2 p}^{\max }=0.6, \bar{\rho}_{p}^{\circ}=7$ we get $\alpha_{1 p 0}^{*} \cong 0.07, \quad \bar{v}_{2 s}^{\max } \cong 10$. As $\alpha_{1 p 0} \geq 0$ a maximum of dependence $\bar{v}_{2 s}\left(\alpha_{1 p 0}\right)$ exists when the condition $\alpha_{20} \leq 2 / 17$. It is obvious that the value $\alpha_{1 p 0}^{*}$ can only be in the range from 0 to $2 \rho_{2}^{\circ} / 17 \rho_{p}^{\circ}$. When $\alpha_{20}>2 / 17$, the velocity $\bar{v}_{2 s}$ with increase $\alpha_{1 p 0}$ monotonically decreases. The results of calculations according to formula (6) below (see Fig. 5).

The non-monotonic behavior of the limit speed is explained by the fact that with increasing $\alpha_{1 p 0}$ increases limit volume fraction of large particles $\alpha_{2 s}$, consequently, the limit size of aggregates $\bar{r}_{2 s}$. Growth $\bar{r}_{2 s}$ leads to an increased rate of sedimentation of aggregates, and magnification $\alpha_{2 s}$ leads to increased influence of straitened sedimentation.

For small values of parameter $q=\theta \alpha_{1 p 0} / \alpha_{20}<<1$ for the deposition rate of the aggregate $\bar{v}_{2 s}$ there are following asymptotic representation

$$
\bar{v}_{2 s} \cong\left(\bar{\rho}_{2}^{\circ}-1\right)\left(1-\alpha_{20}\right)^{4}\left[1-\alpha_{20}+\frac{17}{3}\left(\frac{2}{17}-\alpha_{20}\right) q\right] .
$$

This shows that when $\alpha_{20}<2 / 17$ the speed $\bar{v}_{2 s}$ with increasing $q$ is also growing, whereas when $\alpha_{20}>2 / 17$, in the contrary, it decreases. If $\alpha_{20}=2 / 17$ the sedimentation rate $\bar{v}_{2 s}$ does not depend on a small parameter $q$, it is expressed in a more compact form $\bar{v}_{2 s} \cong\left(\bar{\rho}_{2}^{\circ}-1\right)\left(1-\alpha_{20}\right)^{5}$.

\subsection{Newtonian Motion Mode of the Aggregate}

In other extreme condition corresponding to high Reynolds number of the relative motion of the aggregate (when you can take $C_{\mu}^{\circ} \cong 0.5$ ) for a steady rate of sedimentation we have

$$
\bar{v}_{2 s}=\left[\frac{2\left(\bar{\rho}_{2}^{\circ}-1\right) \bar{r}_{2 s}}{\gamma \psi\left(\alpha_{2 s}\right)}\right]^{1 / 2}, \gamma=\frac{3}{8} \frac{U^{2}}{g r_{20}} .
$$

Similarly to Stokes mode, in this case rate $\bar{v}_{2 s}$ during increase $\alpha_{20}$ monotonically decreases, and with a change $\alpha_{1 p 0}$ behaves non-monotonically and has maximum at the following value of $\alpha_{1 p 0}$

$$
\alpha_{1 p 0}=\alpha_{1 p 0}^{*}=\frac{1}{\theta}\left(\frac{1}{16}-\alpha_{20}\right) .
$$

Limiting volume concentration of the coarse fraction corresponding to this value $\alpha_{1 p 0}^{*}$ is equal to 


$$
\alpha_{2 s}^{*}=\alpha_{20}+\theta \alpha_{1 p 0}^{*}=\frac{1}{16} .
$$

The corresponding maximum rate is calculated by the formula

$$
\bar{v}_{2 s}^{\max } \cong 0.7582\left(\frac{\bar{\rho}_{2}^{\circ}-1}{\gamma}\right)^{1 / 2} \frac{1}{\alpha_{20}^{1 / 6}}
$$

Note that the maximum rate in the Newton mode is achieved at a lower value $\alpha_{1 p 0}$ than in the Stokes regime (compare formulas (7) and (10)).

For small values of $q<<1$ for the sedimentation rate of the aggregate in the Newtonian mode, similar to a Stokes regime, the asymptotic representation is

$$
\bar{v}_{2 s} \cong \beta\left(1-\alpha_{20}\right)^{3 / 2}\left[1-\alpha_{20}+\frac{8}{3}\left(\frac{1}{16}-\alpha_{20}\right) q\right], \quad \beta=\left[\frac{2\left(\bar{\rho}_{2}^{\circ}-1\right)}{\gamma}\right]^{1 / 2},
$$

from which, in particular, follows that with $\alpha_{20}<1 / 16$ the value $\bar{v}_{2 s}$ with growth of $q$ is increased, whereas when $\alpha_{20}>1 / 16$, conversely, decreases. When $\alpha_{20}=1 / 16$ the rate of deposition of aggregates does not depend on a small parameter $q$ and has a simpler form $\bar{v}_{2 s} \cong \beta\left(1-\alpha_{20}\right)^{5 / 2}$.

Comparing formulas (6) and (9), it should be noted that the steady rate (dimensionless) of aggregate sedimentation in the Newtonian regime is proportional to $\bar{r}_{2 s}^{1 / 2}$, whereas it is proportional to $\bar{r}_{2 s}^{2}$ in the Stokes mode. From this we can conclude that in any mode of movement of the aggregate (when the Reynolds number takes not very small and not very large values) $\bar{v}_{2 s} \propto \bar{r}_{2 s}^{\omega}$, where $0.5 \leq \omega \leq 2$. In this case the value $\alpha_{1 p 0}^{*}$ corresponding to the maximum of stationary speed of aggregate satisfies condition

$$
\frac{1}{\theta}\left(\frac{1}{16}-\alpha_{20}\right) \leq \alpha_{1 p 0}^{*} \leq \frac{1}{\theta}\left(\frac{2}{17}-\alpha_{20}\right)
$$

or for limit volume part of coarse particles - the condition

$$
\frac{1}{16} \leq \alpha_{2 s}^{*} \leq \frac{2}{17} \text {. }
$$

Note that the expressions (6), (9) can be used, in particular, for an approximate calculation of the steady rate of sedimentation, respectively, for small and large scale aggregates, and the asymptotic formulas (8), (11) - at low volume concentration of the fine fraction (when $\left.\alpha_{1 p 0}<<\alpha_{20} / \theta\right)$.

\subsection{Numerical Results}

After this there are discussed some results obtained by numerical integration of the system of equations (1) and (2) under the following fixed parameters:

$$
\alpha_{1 p}^{\max }=0.74, \alpha_{2 p}^{\max }=0.6, \bar{\rho}_{p}^{\circ}=7, r_{20}=50 \mu \mathrm{m}, r_{p}=0.05 \mu \mathrm{m},
$$

$T=293 \mathrm{~K}$

The initial volume concentrations of fine and coarse fractions $\alpha_{1 p 0}$ and $\alpha_{20}$ were chosen taking into account the constraints (5) and ranged from 0 to 0.35 and from $10^{-4}$ to 0.2 , respectively.

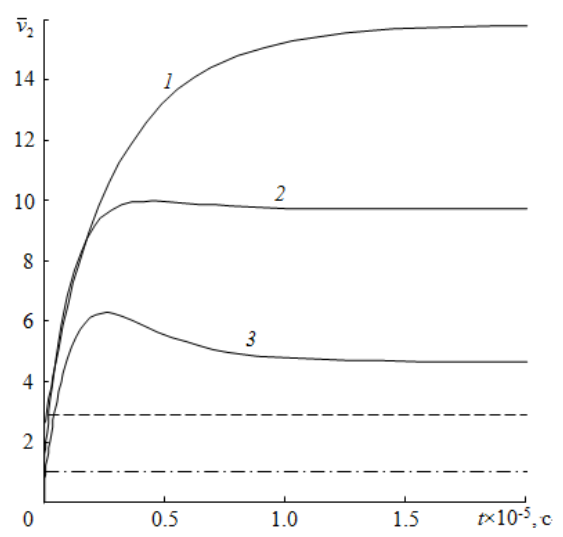

Fig. 2. Dependence of deposition rate of the aggregate at time when $\alpha_{20}$ $=0.001: 1-\alpha_{1 p 0}=0.05,2-0.2,3-0.3$.

Fig. 2 shows time variation of the rate of deposition aggregates $\bar{v}_{2}=-\bar{u}_{2}>0$ for the initial concentration of the coarse fraction $\alpha_{20}=0.001$. Curves $1-3$ correspond to different initial volume content of the fine fraction $\alpha_{1 p 0}$ $=0.05$ (curve 1), 0.2 (2), 0.3 (3). For comparison, a dotdashed line shows the steady-state speed of a large particle in the absence of inter-fractional coagulation (for $\alpha_{1 p 0}$ $=0.2)$, and the dashed line - without the fine fraction $\left(\alpha_{1 p 0}\right.$ $=0)$. It is evident that depending on the volumetric concentration of the fine fraction the deposition rate of the aggregate behaves differently. In non-highly concentrated suspension aggregate speed gradually increases, reaching its steady-state value for a long time (curve 1 ). Whereas in the process of sedimentation slurry concentrated coarse fraction can be divided into two stages (curves 2, 3). In the first stage rate increases because larger particles to increase its mass by addition of fine particles, and the second stage where it eventually reaches a certain size, the sedimentation rate decreases due to the effect of constraint. In this range of the concentration of the initial volume fraction of the sedimentation rate of small aggregate in the presence of the coagulation process is substantially higher than in its absence. However, we note that an increase $\alpha_{1 p 0}$ rate of deposition of the aggregate due to the influence of straitened sedimentation effect may be less than in the pure liquid. The effect of the steady rate $\alpha_{1 p 0}$ on a large particle in a suspension is discussed below (see Fig. 5). 


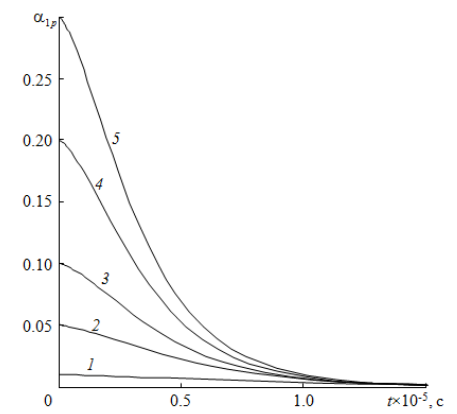

Fig. 3. Dependence of the volume concentration of the fine fraction of the suspension from time when $\alpha_{20}=0.001: 1-\alpha_{1 p 0}=0.01,2-0.05,3-0.1,4$ $-0.2,5-0.3$.

Fig. 3 shows the variation in time of the volume concentration of fines in its various initial values $\alpha_{1 p 0}$ $=0.01$ (curve 1), 0.05 (2), 0.1 (3), 0.2 (4), 0.3 (5). Value $\alpha_{20}$ is the same as in Fig. 2. It is seen that at time $10^{5} \mathrm{sec}$ despite the different speeds of aggregates at different $\alpha_{1 p 0}$ (see Fig. 2) the volume concentration of the fine fraction decreases significantly.

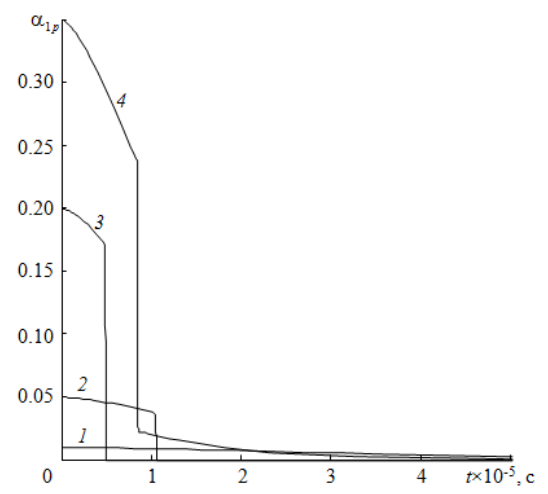

Fig. 4. The same as in Fig. 3 but with $\alpha_{20}=0.0001: 1-\alpha_{1 p 0}=0.01,2-$ $0.05,3-0.2,4-0.35$.

Fig. 4 illustrates the behavior at lower values (compared with the previous case) the initial value of the volume concentration of the coarse fraction $\alpha_{20}=0.0001$. Curves 1 4 correspond to $\alpha_{1 p 0}=0.01$ (curve 1), 0.05 (2), 0.2 (3), 0.35 (4). It can be seen that not very concentrated slurry volume fraction of fines due to coagulation is reduced as in the previous case, slowly enough (curve 1). While more concentrated slurry coagulation of fine and coarse fractions occurs slowly at first, then, unlike the previous case, in a relatively short time the fine fraction is almost completely converted into aggregates (curves 2-4). The latter is due to the fact that reducing the volume content of coarse particles (fixed size) of the number density of the slurry is reduced. Consequently, by joining aggregates of fine particles, compared with the previous case, reach the larger sizes to which the flow regime changes from a viscous to potential. Thus, in accordance with formula (3) the intensity of transfer of fine fraction to the coarse fraction significantly larger, as in the viscous regime it is proportional to $\delta^{2}$, and in the potential regime - to $\delta(\delta<<1)$.

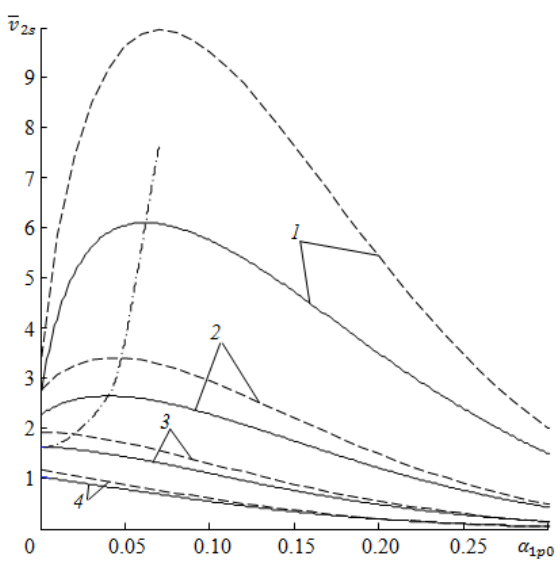

Fig. 5. Dependence of stationary velocity of aggregate on volume concentration of fine particle fraction for different $\alpha_{20}: 1-\alpha_{20}=0.01,2$ - 0.05, 3 - 2/17, $4-0.2$.

Fig. 5 shows the sedimentation rate of limit aggregate $\bar{v}_{2 s}$ from an initial concentration of fines in suspension at different initial concentrations of a large volume fraction $\alpha_{20}=0.01$ (curve 1), 0.05 (2), 2/17 (3), 0.2 (4). The solid curves correspond to the numerical integration of the system (1), (2), and dashed curves are based on the formula (6) and the corresponding Stokes regime of movement of the aggregate. Calculations show that, in general, similar to Stokes and Newtonian regimes the dependence $\bar{v}_{2 s}\left(\alpha_{1 p 0}\right)$ from the values $\alpha_{20}$ behaves qualitatively different. For example, at about $\alpha_{20}<\sim 2 / 17$ considering dependence varies non-monotonically (curves 1,2 ) and passes through a maximum, whereas with $\alpha_{20}>\sim 2 / 17$ it monotonically decreases with increasing $\alpha_{1 p 0}$ (curves 3 and 4). Moreover, with a decrease $\alpha_{20}$ the maximum point shifts to higher $\alpha_{1 p 0}$ (as evidenced by the behavior of the dot-dashed line). Note that with increasing initial volume content of the coarse fraction $\alpha_{20}$ difference in behavior $\bar{v}_{2 s}\left(\alpha_{1 p 0}\right)$ according to the Stokes and general cases decreases. This is understandable, because with the increase $\alpha_{20}$ due to the effects of constraint deposition rate of the aggregate falls, and the conditions for movement close to Stokes regime.

\subsection{Conclusions}

Thus, in the model there are analyzed some features of the dynamics of aggregates in a spatially homogeneous coagulating suspension with the straitened sedimentation effect. Note the given work is theoretical. The results of the analysis and the resulting approximate formulas can be used for estimation and calculation the general parameters of sediment suspension in the presence of the coagulation process. 


\section{Acknowledgments}

This work was supported by a grants (№ 0750/GF and №1117/GF) of the Ministry of Education and Science of the Republic of Kazakhstan.

\section{References}

[1] T.R. Camp, Floc volume concentration, J. Amer. Water Works Assoc.60 (1962) № 6. P. 656.

[2] N.B. Uriev and A.A. Potanin, Fluidity of suspensions and powders, Moscou: Chemistry, 1992. (in Russian)

[3] L.V. Ravitchev, A.V. Bespalov and V.J. Loginov, Simulation of viscous properties of concentrated suspensions, Theoretical Found. of Chemical Engineering, 2008. V. 24. №3. P. 326 .

[4] M.P. Volarovich and N.V. Churaev, Investigation of the degree of dispersion of peat, Proceedings of the Moscow Institute of peat, Moscow, 1955. Issue 3. P. 33. (in Russian)

[5] B.M. Dolgonosov, The kinetics of coagulationfragmentation and the equilibrium spectrum of aggregates in moving suspensions, Theoretical Found. of Chemical Engineering, 2001. V. 35. № 5. P. 465.

[6] V.A. Galkin, I.V. Galkin, D.Y. Osetsky, D.A. Rizhikov and A.V. Galkin, Mathematical modeling of sintering of powder materials and the growth of Aggregates, Proceedings of the regional competition of research projects in the field of natural sciences, Kaluga, 2007. Issue 11. P. 42. (in Russian)

[7] B.M. Dolgonosov, The kinetics of the coagulating sedimentation suspension, Theoretical Found. of Chemical Engineering, 2005. V. 39. № 6. P. 673.

[8] B.I. Brounshteyn and V.V. Schogolev, Hydrodynamics, mass and heat transfer in the column apparatuses, Moscou: Chemistry, 1980. (in Russian)

[9] J. Happel and H. Brenner, Hydrodynamics at low Reynolds numbers, Springer-Verlag, 1976.

[10] R.I. Nigmatulin, Fundamentals of mechanics of heterogeneous media, Moscow: Nauka, 1978. (in Russian)

[11] V.M. Voloshchuk and Y.S. Sedunov, Coagulation processes in disperse Systems, Leningrad: Gidrometeoizdat, 1975. (in Russian)

[12] Theory and technology of flotation, Ed. Bogdanov O.S., Moscow: Nedra, 1990. (in Russian)

[13] R.H. Yoon and G.H. Luttrell, The effect of bubble size on fine particle flotation, Miner. Process. Extr. Metal. Rev. 1989. № 5. P. 101.

[14] G.K. Batchelor, Sedimentation in a dilute dispersion of spheres, J. Fluid Mech. 1972. V. 52. P. 245.

[15] A.M. Golovin and V. Chizhov, Calculation of the deposition rate of a homogeneous suspension, J. Appl. Math. and Mechanics, 1978. V. 42. № 1. P.105.

[16] M. Mooney, The viscosity of concentrated suspensions of spherical particles, J. Coll. Sci. 1951. V. 6. №2. P. 162.

[17] T.L. Smith and C.A. Bruce, The viscosity of concentrated suspensions, J. Coll. Interface Sci. 1979. V. 72. № 1. P. 13. 